\title{
Increases in moist-convective updraught velocities with warming in radiative-convective equilibrium
}

\author{
Martin S. Singh ${ }^{\mathrm{a} \star}$ and Paul A. O'Gorman ${ }^{\mathrm{b}}$ \\ ${ }^{a}$ Department of Earth and Planetary Sciences, Harvard University, Cambridge, MA, USA \\ ${ }^{\mathrm{b}}$ Department of Earth, Atmospheric and Planetary Sciences, Massachusetts Institute of Technology, Cambridge, MA, USA \\ ${ }^{\star}$ Correspondence to: M. S. Singh, Department of Earth and Planetary Sciences, Harvard University, 20 Oxford Street, Cambridge, \\ MA 02138, USA. E-mail: martinsingh@fas.harvard.edu
}

\begin{abstract}
The scaling of updraught velocities over a wide range of surface temperatures is investigated in simulations of radiative-convective equilibrium with a cloud-system resolving model. The updraught velocities increase with warming, with the largest fractional increases occurring in the upper troposphere and for the highest percentile updraughts. A plume model approximately reproduces the increases in updraught velocities if the plume environment is prescribed as the mean profile in each simulation while holding the entrainment and microphysical assumptions fixed. Convective available potential energy (CAPE) also increases with warming in the simulations but at a much faster fractional rate when compared with the square of the updraught velocities. This discrepancy is investigated with a two-plume model in which a weakly entraining plume represents the most intense updraughts, and the environment is assumed to adjust so that a more strongly entraining plume has negligible buoyancy. The two-plume model suggests that updraught velocities increase with warming at a lower fractional rate than implied by the CAPE because of the influence of entrainment on both the mean stratification and the updraughts themselves.
\end{abstract}

Key Words: moist convection; updraught velocities; radiative-convective equilibrium; cloud-system resolving model; climate change; deep convection

Received 11 November 2014; Revised 11 February 2015; Accepted 22 April 2015; Published online in Wiley Online Library 04 June 2015

\section{Introduction}

Moist-convective updraught velocities are a key determinant of the precipitation rate associated with convective clouds (e.g. Muller et al., 2011) and the lightning frequency in thunderstorms (e.g. Baker et al., 1995), and they also influence cloud microphysical properties (e.g. Del Genio et al., 2005). While general circulation models (GCMs) are typically run at resolutions too coarse to resolve individual convective clouds, they do predict changes to large-scale fields that imply increases in convective available potential energy (CAPE) with warming (Sobel and Camargo, 2011; Fasullo, 2012), suggesting the possibility for future increases in updraught velocity (Del Genio et al., 2007). However, the relationship between large-scale fields and convective updraught velocities is not straightforward, and it is unclear to what extent quantities such as the CAPE may be used as a proxy for the updraught velocity.

An alternative approach is to use cloud-system resolving models (CRMs) to study the problem in the idealized setting of radiative-convective equilibrium (RCE). Recent studies have found that both updraught velocities and CAPE increase as the atmosphere warms in simulations of RCE (Romps, 2011; Muller et al., 2011). Singh and O'Gorman (2013, hereafter SO13) attributed this CAPE increase to the effect of entrainment in altering the mean lapse rate from moist adiabatic, an effect that becomes more pronounced at higher temperatures. They introduced a zero-buoyancy plume model in which the convective ensemble is represented by a bulk entraining plume that is assumed to remain neutrally buoyant with respect to the environment. As the atmosphere warms, the relative humidity does not vary greatly, and the saturation deficit of the troposphere increases. The greater saturation deficit in a warmer atmosphere implies that a given amount of entrainment has a larger effect in reducing the plume temperature which, by the zero-buoyancy assumption, alters the environmental lapse rate further away from that of a moist adiabat.

The above argument was used by SO13 to account for the changes in mean stratification and CAPE with warming in simulations of RCE. But the neglect of the cloud buoyancy precludes its use to reason about updraught velocity. Moreover, the fractional rate of increase of the vertically integrated cloud buoyancy (the buoyancy integral) was found to be considerably smaller than that of the CAPE. Here we build on the work of SO13, and seek to explain the increases in updraught velocities and cloud buoyancy with warming in RCE, and to understand their relationship, if any, to the increase in CAPE.

Previous studies have attempted to construct theories of convective updraught velocity using constraints on frictional dissipation derived from the entropy budget (Emanuel and Bister, 1996; Rennó and Ingersoll, 1996). However, as pointed 
out by Pauluis and Held (2002), moist processes are the dominant irreversible entropy source in the atmosphere. This complicates efforts to constrain the frictional dissipation rate and suggests cloud and precipitation microphysics may play a role in determining updraught velocities in moist-convective clouds. Indeed, Parodi and Emanuel (2009) found that the fall speed of hydrometeors is an important factor in determining convective updraught velocities in RCE; a low fall speed results in clouds with higher condensed water contents and thus reduced buoyancy via the water loading effect. Hydrometeor fall speeds, and microphysical processes more generally, are sensitive to temperature changes (Singh and O'Gorman, 2014) and thus may contribute to changes in updraught velocity as the atmosphere warms.

In this study, we consider CRM simulations of RCE similar to those of SO13 in which the surface temperature is varied over a wide range. Consistent with previous studies (Muller et al., 2011; Romps, 2011), we find increases in updraught velocities with warming, particularly in the upper troposphere. We seek to understand these increases through a series of models of a convective ensemble based on entraining plumes. In developing such models, we are adopting a simplified view of moist convection in which we neglect vertical mixing within clouds (Paluch, 1979), the episodic nature of cloud entrainment (Romps and Kuang, 2010b), and buoyancy sorting (Raymond and Blyth, 1986). Nevertheless, plume-based models have had considerable success as the basis of convection parametrizations (e.g. Arakawa and Schubert, 1974; Tiedtke, 1989), and they have been argued to be a reasonable starting point in modelling an ensemble of convective clouds (Lin and Arakawa, 1997; Kuang and Bretherton, 2006).

We first investigate the increases in updraught velocities using a spectrum of entraining plumes with the environment represented as the mean profiles in the simulations. The results suggest that changes to the mean stratification of the troposphere play a leading role in governing the response of the simulated updraught velocities to warming, despite the lack of proportionality between CAPE and the buoyancy integral. We then investigate the relative scaling of the buoyancy integral and CAPE using a generalization of the zero-buoyancy plume model introduced in SO13. We extend this model to include two plumes, with one plume representing the most intense updraughts and the second plume representing the bulk of the convective mass flux. As in SO13, the environmental lapse rate is derived by assuming the bulk plume is neutrally buoyant with respect to the environment. Based on the simulations and both the spectrum of plumes and the two-plume model, we argue that the lower fractional rate of increase of the buoyancy integral with temperature compared to that of CAPE is a result of how entrainment affects both the mean stratification and the buoyancy of the most intense updraughts. On the other hand, the influence of hydrometeor fall speeds on updraught velocities is relatively limited.

The rest of the article is organized as follows: we first describe the simulations (section 2) and the results for cloud buoyancy and updraught velocities (section 3). We then interpret the scaling of cloud buoyancy and updraught velocities using an entrainingplume model in which the plume environment is taken from the simulations (section 4), before incorporating the environmental lapse rate into the plume solution via a generalization of the zero-buoyancy plume model (section 5). Finally, we investigate the effect of changing hydrometeor fall speeds on the scaling of updraught velocities with warming (section 6) before recapping the main findings of this work and their potential implications for a warming climate (section 7).

\section{Simulations of radiative-convective equilibrium}

We conduct simulations of RCE in a doubly periodic domain using a model similar to version 16 of the Bryan cloud model (Bryan and Fritsch, 2002), with minor alterations to improve mass and energy conservation as described in Singh (2014). The simulations are similar to those used in $\mathrm{SO} 13$ and identical to the high-resolution simulations used in Singh and O'Gorman (2014), but we describe them below for completeness.

The model is fully compressible and non-hydrostatic, and it uses a split-explicit time-stepping scheme based on Wicker and Skamarock (2002). A sixth-order spatial-differencing scheme is used, and it is coupled with a sixth-order hyper-diffusion scheme for numerical stability. The effects of subgrid-scale motions are parametrized via a Smagorinsky turbulence scheme with separate diffusion coefficients for the vertical and horizontal directions (Bryan and Rotunno, 2009). Fluxes of sensible heat, moisture and momentum from the surface are calculated using bulk aerodynamic formulae, with turbulent exchange coefficients evaluated based on Monin-Obukov similarity theory. The microphysics scheme is a single-moment scheme based on Lin et al. (1983), as modified by Braun and Tao (2000). It includes six prognostic water species and is identical to the 'Lin-hail' scheme described in Singh and O'Gorman (2014). A full radiative transfer scheme is used, but there is no diurnal cycle; the solar flux is assumed to be $390 \mathrm{~W} \mathrm{~m}^{-2}$ in all simulations, and the zenith angle is fixed at $43^{\circ}$. Simulations are run with identical ozone profiles typical of the tropical atmosphere with a maximum in mixing ratio near $10 \mathrm{hPa}$. Each simulation includes 64 vertical levels, with the model top set at $26 \mathrm{~km}$. Rayleigh damping is applied to the velocity fields in the upper $2 \mathrm{~km}$ to prevent gravity-wave reflection.

Simulations of RCE with different imposed $\mathrm{CO}_{2}$ concentrations in the range $1-640 \mathrm{ppmv}$ are first conducted over a slab ocean until statistical equilibrium is reached. The slab ocean has a uniform temperature and a depth of $1 \mathrm{~m}$. While this is considerably shallower than the ocean mixed layer, the uniform temperature of the slab ensures that it responds only to the domain-integrated net flux of energy at the surface, and that it is insensitive to cloud-scale anomalies in surface radiative and turbulent fluxes (Romps, 2011). Because of the long integration time needed to reach equilibrium (Cronin and Emanuel, 2013), the slab-ocean simulations are run in a low-resolution, smalldomain configuration with a horizontal grid-spacing of $2 \mathrm{~km}$ and a domain $80 \times 80 \mathrm{~km}$ in size. The slab-ocean simulations equilibrate with sea-surface temperatures (SSTs) in the range $281-311 \mathrm{~K}$ (Figure 1). Here, equilibration is defined as occurring when the magnitude of the ocean temperature trend for the last 50 days of the simulation is less than $1 \mathrm{Kyear}^{-1}$.

The low-resolution simulations are then repeated at higher horizontal resolution $(0.5 \mathrm{~km}$ grid spacing) and on a larger domain $(160 \times 160 \mathrm{~km})$ with a fixed-SST lower-boundary condition, taken as the equilibrated value of the SST in the corresponding low-resolution, slab-ocean simulation. These high-resolution simulations are initialized with the equilibrium temperature and humidity profiles of the slab-ocean simulations and run for 30 days, with statistics collected at hourly intervals over the last 10 days.

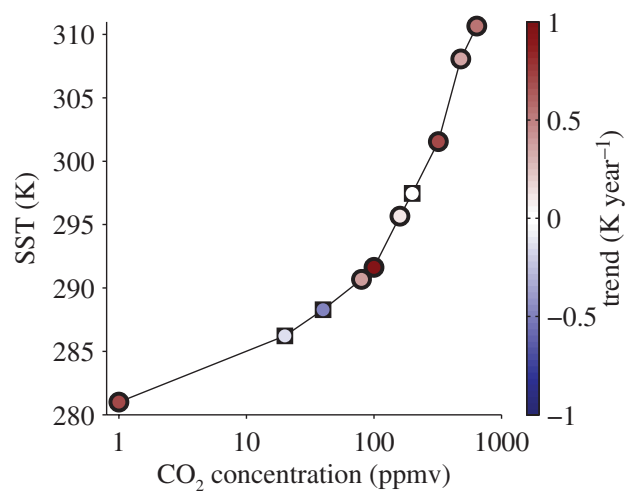

Figure 1. Equilibrium SST for the slab-ocean simulations as a function of the imposed $\mathrm{CO}_{2}$ concentration. The equilibrium is defined as the mean over the last 50 days of each simulation. Symbols and colours represent the linear trend in slab-ocean temperature over this period; simulations with positive (negative) trends are plotted as circles (squares). 


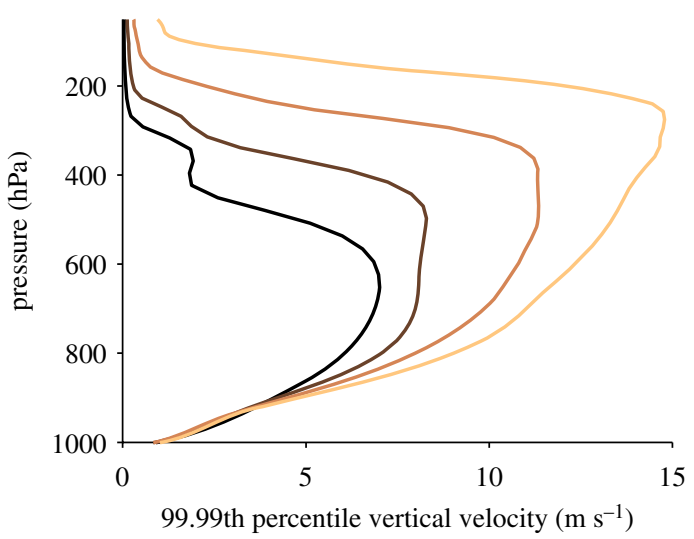

Figure 2. The 99.99th percentile of vertical velocity in high-resolution simulations with SSTs of 281 (black), 291, 302 and $311 \mathrm{~K}$ (orange). The 99.99th percentile is calculated at each level in each simulation, and the results are plotted as vertical profiles.

The fixed-SST boundary condition allows the high-resolution simulations to come to equilibrium more rapidly than the slabocean simulations, but it also implies that the high-resolution simulations are not energetically closed. While they have the same $\mathrm{CO}_{2}$ concentration as the corresponding slab-ocean simulation, top-of-atmosphere energy imbalances of the order of a few $\mathrm{W} \mathrm{m}^{-2}$ remain in the high-resolution simulations even at equilibrium. These small imbalances are not expected to strongly affect the updraught velocities or cloud buoyancies that are our primary interest here, and for the rest of this article we focus on results from the high-resolution simulations, which are better able to resolve cloud updraughts. Our conclusions would not be altered significantly by considering the low-resolution, slab-ocean simulations.

\section{Updraught velocities and cloud buoyancy}

Figure 2 shows vertical profiles of the 99.99th percentile of vertical velocity (calculated at each level including all points) in a number of the simulations. This measure of the vertical velocity increases as the surface warms, although the increase is not uniform with height, but rather is more pronounced in the upper troposphere. The peak value of the profiles shown in Figure 2, which we denote $w_{\max }$, increases with warming by roughly a factor of 2 over the range of surface temperatures simulated. The fractional increase at a fixed pressure in the lower troposphere is somewhat weaker, while at levels below $900 \mathrm{hPa}$ there is almost no change. The changes with warming also depend on the percentile chosen. The peak value of a higher percentile (99.999th) vertical velocity profile increases by a factor of 2.5 over the range of surface temperatures simulated, while for a lower percentile (99.9th) the increase is only by a factor of 1.2. We focus on a relatively high percentile (the 99.99th percentile) because intense convective updraughts are associated with a number of impacts and because high percentiles of the vertical velocity are found to be relatively straightforward to analyze (results for updraughts averaged over the convective cores are also discussed later).

To help understand the increases in updraught velocities with warming, we consider various measures of buoyancy in the simulations. We first consider the buoyancy of an undilute parcel lifted adiabatically from near the surface. As pointed out by SO13, the mean temperature profiles in RCE systematically deviate from those of a moist adiabat (Figure 3(a)), implying that the buoyancy of an undilute parcel increases with warming. An integral measure of this undilute buoyancy is the CAPE; here we calculate the CAPE as the integral of the positive buoyancy of a reversible and adiabatic parcel ascent up to its level of neutral buoyancy. For each simulation, the parcel buoyancy is taken with respect to the domain- and time-mean virtual-temperature profile, and the parcel is initialized with the mean temperature and specific humidity at the lowest model level. To be consistent
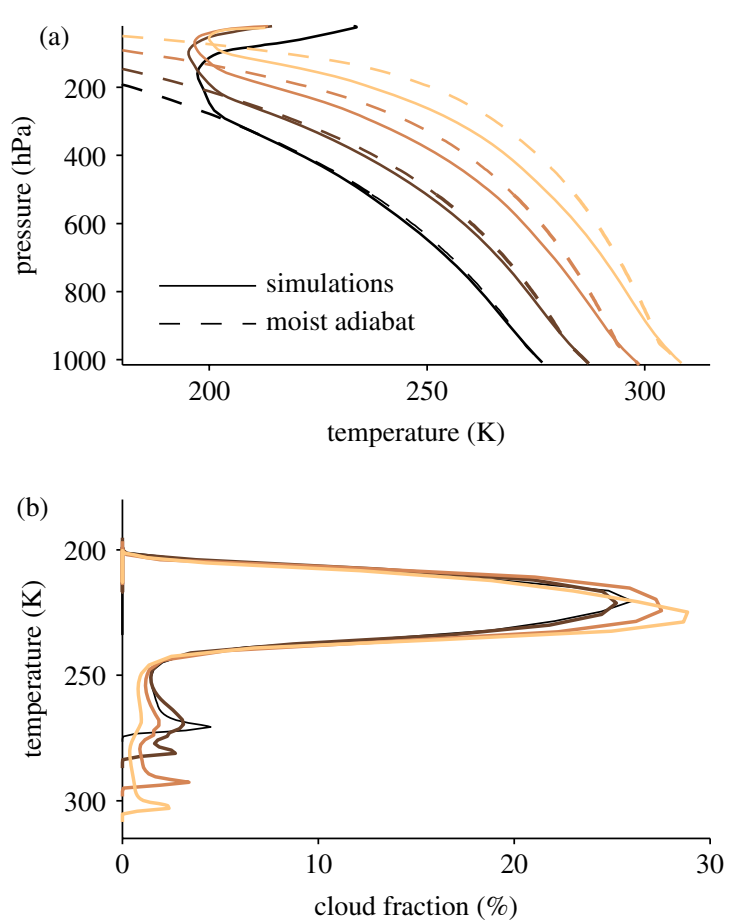

Figure 3. Properties of high-resolution simulations with SSTs of 281 (black), 291, 302 and $311 \mathrm{~K}$ (orange). (a) Domain- and time-mean temperature (solid), and the temperature of a reversible and adiabatic parcel ascent initialized with the mean properties at the lowest model level in the corresponding simulation (dashed), plotted as a function of pressure. (b) Mean cloud fraction plotted against mean temperature; cloudy grid points are defined as those in which the mixing ratio of non-precipitating condensate is greater than $0.01 \mathrm{~g} \mathrm{~kg}^{-1}$.

with the thermodynamic formulation in the CRM, the fraction of condensate in the parcel comprised of ice is assumed to increase linearly as the temperature decreases from 273.15 to $233.15 \mathrm{~K}$. As found in SO13, the CAPE increases greatly with warming; its value in the warmest simulation is roughly a factor of 60 greater than its value in the coldest simulation (Figure 4(a)).

The CAPE is considerably larger in magnitude than a similar measure of available potential energy based on the simulated buoyancy of clouds. We define the buoyancy integral as the vertical integral of the 99.99th percentile of buoyancy at each level in the simulations. The buoyancy includes the water loading effect of both precipitating and non-precipitating condensates, and the integral is taken from the surface to a nominal cloud top, defined here as the level at which the mean temperature in the simulation equals $220 \mathrm{~K}$. This level was chosen because it provides a good approximation to the detrainment level for deep convective clouds, as measured by the maximum in mean cloud fraction in the simulations (Figure $3(\mathrm{~b})$ ). This approximation is consistent with the 'fixed anvil temperature' hypothesis introduced by Hartmann and Larson (2002) and corresponds to an upward shift of atmospheric properties with warming in the middle and upper troposphere (see also Tompkins and Craig, 1999; Kuang and Hartmann, 2007; Singh and O'Gorman, 2012; Khairoutdinov and Emanuel, 2013). Like the CAPE, the buoyancy integral increases with warming, but it is nearly an order of magnitude smaller than the CAPE, and its fractional rate of increase is considerably lower than that of the CAPE; it increases by a factor of roughly 6 over the range of surface temperatures simulated, compared to 60 for the CAPE.

As expected from the application of a simple force balance (cf. Eq. (6)), the buoyancy integral is tightly coupled to the peak kinetic energy per unit mass of the updraught profiles shown in Figure 2; a roughly linear relationship exists between the buoyancy integral and $w_{\max }^{2} / 2$ across the simulations (Figure 4(b), circles).

A similar, albeit less linear, relationship holds for alternative measures of the peak kinetic energy and buoyancy integral based on conditional-mean profiles for points within 'convective cores' (Figure 4(b), crosses). Here, convective cores are comprised of 

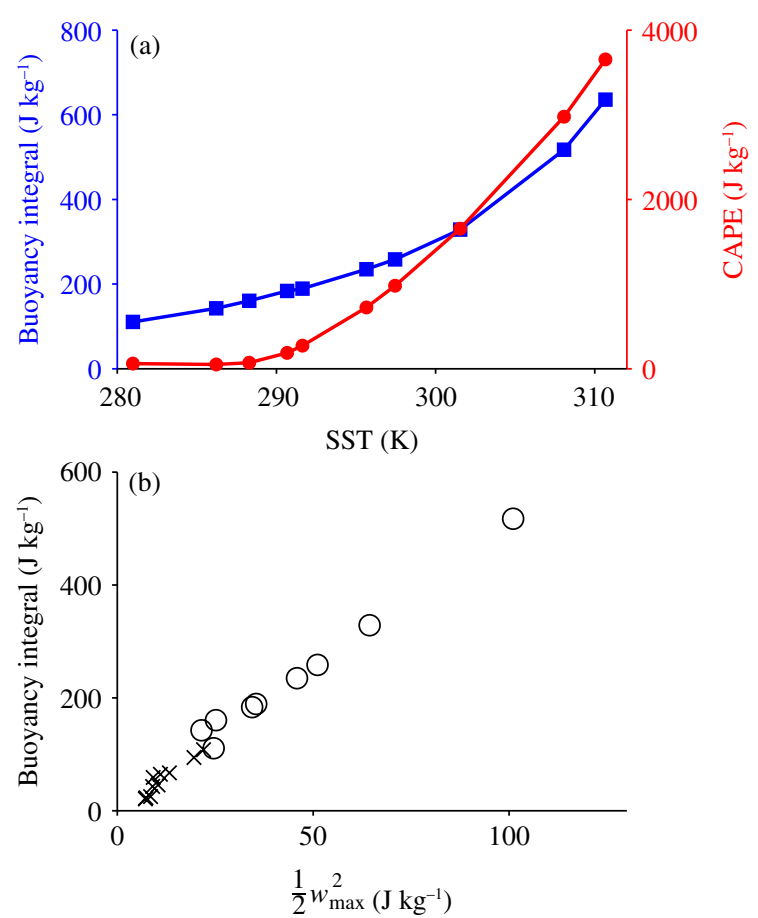

Figure 4. (a) Reversible CAPE (red line and circles, right axis) and buoyancy integral for the 99.99th percentile (blue line and squares, left axis) in highresolution simulations as a function of SST. (b) Relationship between peak kinetic energy $w_{\max }^{2} / 2$ and buoyancy integral (both for the 99.99th percentile) in the high-resolution simulations (circles). In (b) crosses denote alternate measures of the buoyancy integral and peak kinetic energy calculated from a conditional mean over grid points within convective cores (see text for details).

all grid points in which the mixing ratio of non-precpitating condensate is greater than $0.01 \mathrm{~g} \mathrm{~kg}^{-1}$ and the upward velocity is greater than $2 \mathrm{~m} \mathrm{~s}^{-1}$. The buoyancy integral in this case is taken as the integral of the conditional-mean buoyancy profile over all levels where it is defined, and the peak updraught is taken as the maximum in the conditional-mean vertical velocity profile. This measure of the peak updraught gives smaller values than $w_{\max }$, and it increases with warming at a somewhat lower fractional rate than that of $w_{\max }$.

In summary, we have shown that the updraught velocities increase with warming in the RCE simulations, with the largest fractional increases occurring for the most intense updraughts. Additionally, the buoyancy integral, while being a key determinant of the peak updraught velocity, does not scale with a similar quantity based on an undilute parcel ascent, the CAPE. In the next two sections we seek to understand these results through the conceptual framework of a set of entraining plumes.

\section{Spectrum of plumes with given environment}

We first consider the representation of a convective cloud ensemble by a spectrum of entraining plumes with a range of entrainment rates, and with the environment and lower-boundary conditions taken from the simulations.

\subsection{Calculation of plume properties}

The plume model is written in terms of the vertical profile of moist static energy $h$ and total water mass fraction $q_{\mathrm{t}}$ as

$$
\begin{aligned}
\frac{\mathrm{d} h}{\mathrm{~d} z} & =-\epsilon\left(h-h_{\mathrm{e}}\right), \\
\frac{\mathrm{d} q_{\mathrm{t}}}{\mathrm{d} z} & =-\epsilon\left(q_{\mathrm{t}}-q_{\mathrm{ve}}\right) .
\end{aligned}
$$

Here, $\epsilon$ is the height-independent entrainment rate, $q_{\mathrm{v}}$ is the mass fraction of water vapour (specific humidity), $z$ is height, the subscript e refers to properties of the environment, and we have assumed no precipitation fallout. The moist static energy (MSE) is defined by

$$
h=c_{\mathrm{p}}\left(T-T_{0}\right)+g z+L_{\mathrm{v}} q_{\mathrm{v}}-L_{\mathrm{f}} q_{\mathrm{i}}
$$

where $c_{\mathrm{p}}$ is the isobaric specific heat capacity of moist air, $T$ is the temperature, $T_{0}=273.15 \mathrm{~K}, g$ is the gravitational acceleration, $L_{\mathrm{v}}$ and $L_{\mathrm{f}}$ are the latent heats of vaporization and freezing evaluated at $T_{0}$, and $q_{\mathrm{i}}$ is the mass fraction of ice.

For each simulation, we calculate vertical profiles of MSE and total water mass fraction for 38 plumes with different entrainment rates ranging between 0 and $1.5 \mathrm{~km}^{-1}$. We integrate the plume equations (1) and (2) upwards from the lowest model level assuming no fallout of condensate, and assuming the environmental temperature and specific humidity are equal to those of the time and domain mean in the corresponding simulation. The integrations are performed using forward differences on a grid with vertical spacing of $50 \mathrm{~m}$; the environmental profile is interpolated linearly in height from the model levels to this grid (further details of the method of solution may be found in SO13).

To account for the effect of boundary-layer inhomogeneity, we set the temperature and specific humidity of the plumes at the lowest model level to be equal to one standard deviation above the mean in the simulations. Specifically, the plume temperature $T_{\mathrm{b}}$ and specific humidity $q_{\mathrm{b}}$ at the lower boundary are given by

$$
\begin{aligned}
& T_{\mathrm{b}}=\bar{T}+\sigma_{T}, \\
& q_{\mathrm{b}}=\bar{q}+\sigma_{q},
\end{aligned}
$$

where $\bar{T}$ and $\bar{q}$ are the mean temperature and specific humidity, respectively, and $\sigma_{T}$ and $\sigma_{q}$ are the standard deviations of temperature and specific humidity, respectively, all at the lowest model level of the corresponding simulation. A single value for the properties of the plumes at the lowest model level is adopted for simplicity; it is shown below that this is a reasonable approximation for simulations of RCE.

As mentioned above, we assume no precipitation fallout in the plume calculations. The fraction $f$ of condensate consisting of ice is assumed to increase linearly as the temperature decreases from 273.15 to $233.15 \mathrm{~K}$. The saturation vapour pressure $e^{*}$ is then taken to be

$$
e^{*}=(1-f) e_{1}^{*}+f e_{\mathrm{i}}^{*},
$$

where $e_{1}^{*}$ and $e_{i}^{*}$ are the saturation vapour pressures over liquid and solid, respectively. This form of the saturation vapour pressure is consistent with that used in the CRM simulations, and no supersaturation is allowed. With these microphysical assumptions and the profiles of $h$ and $q_{\mathrm{t}}$, we can calculate the temperature, buoyancy, and condensed water content of the plumes.

Finally, the vertical velocity within the plumes, $w(z)$, is calculated using a simple vertical velocity equation given by

$$
\frac{1}{2} \frac{\partial w^{2}}{\partial z}=a B-b \epsilon w^{2},
$$

where $w$ is initialized at zero at the lowest model level, and $B$ is the buoyancy. A number of values for $a$ and $b$ have been suggested in the literature (de Roode et al., 2012, give a review); here we follow Bretherton et al. (2004) by setting $a=1$ and $b=2$.

\subsection{Results}

We compare the plume spectrum to the simulations by examining the distribution of air parcel properties in the MSE-height plane. We calculate the cumulative distribution function of MSE at each level based on hourly snapshots from the simulations. Following Kuang and Bretherton (2006), we define the effective entrainment rate at a given point in the MSE-height plane as the value of 

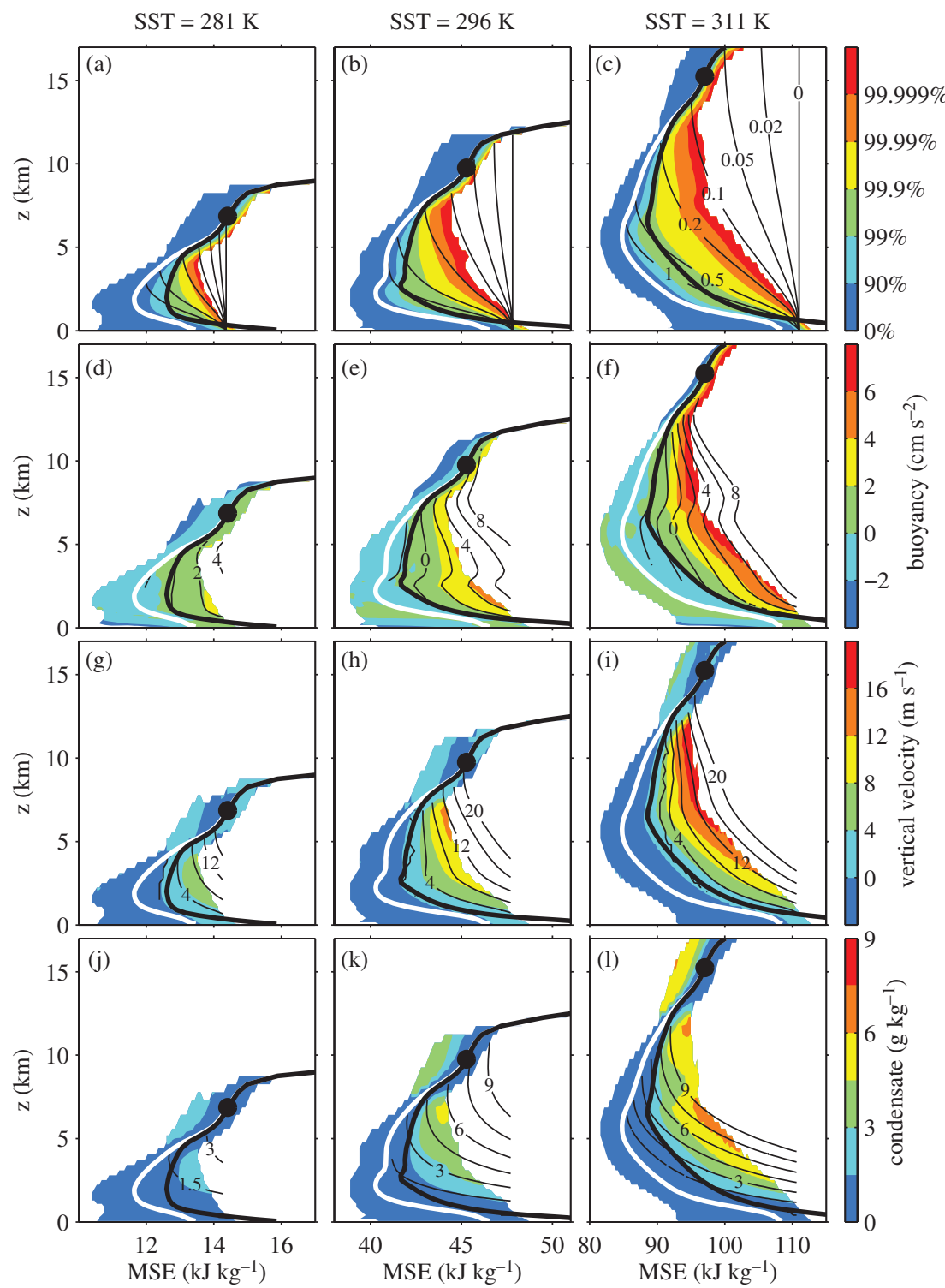

Figure 5. Statistics as a function of MSE and height in three high-resolution simulations of RCE with SSTs of (a,d,g,j) 281 K, (b,e,h,k) $296 \mathrm{~K}$ and (c,f,i,l) $311 \mathrm{~K}$. All panels show the domain- and time-mean MSE (white line) and saturated MSE (thick black line) profiles, and the black dots represent the level at which the mean temperature is equal to $220 \mathrm{~K}$. $(\mathrm{a}-\mathrm{c})$ Cumulative distribution function of moist static energy at each level (colours) and profiles of moist static energy for plume calculations with entrainment rates as given in (c) in units of $\mathrm{km}^{-1}$ (thin black lines). Lower panels show distributions of (d,e,f) buoyancy, (g,h,i) vertical velocity, and $(\mathrm{j}, \mathrm{k}, \mathrm{l})$ total condensate binned by MSE for the simulations (colours) and plume spectrum (thin black contours, units of contour values are as shown on the colour bar). For the simulations, the total condensate includes both precipitating and non-precipitating condensed water species.

$\epsilon$ of the plume passing through that point (see also Pauluis and Mrowiec, 2013). We may then associate different parts of the MSE distribution with different effective entrainment rates (Figure 5(a)-(c)).

In the troposphere, most air parcels in the simulations have MSE values relatively close to that of the mean (white lines), while a small fraction have values of MSE greater than the saturation MSE of the mean profile (thick black lines), corresponding roughly to parcels that are within clouds. Among these cloudy parcels, a range of effective entrainment rates exist, but, consistent with our plume interpretation, the effective entrainment rate for a given percentile of MSE does not vary greatly with height. For example, the 99.999th percentile of MSE has an effective entrainment rate of between 0.1 and $0.2 \mathrm{~km}^{-1}$ over a large portion of the troposphere in all the simulations shown. This high percentile is exceeded by roughly 250 points at each level, and it represents the air parcels with close to the lowest effective entrainment rates at a given level (we show contours up to the 99.9999th percentile). The relatively high value of the effective entrainment rate for the highest percentiles of MSE that we can robustly diagnose implies that undilute ascent from the boundary layer is virtually absent in the simulations, consistent with the results presented in the previous section showing that the buoyancy integral is substantially smaller than the CAPE. Previous CRM studies of deep convection have also found that air parcels rising from the boundary layer to the troposphere undergo considerable mixing with their environment (e.g. Romps and Kuang, 2010a), although some evidence of the rare occurrence of undilute parcels in RCE has been found by Pauluis and Mrowiec (2013).

In the lowest few hundred metres of the atmosphere, the MSE distribution has negative skewness, and a long tail toward low MSE, reflecting the effect of cold pools on the boundary-layer temperature and humidity. In this region, the MSE distribution has a sharp cut-off at high MSE values, suggesting that the variation of updraught properties in the boundary layer is relatively small compared to that in the middle troposphere. Kuang and Bretherton (2006) also found relatively tight distributions of cloud-base properties in shallow and weakly precipitating convection, and Romps and Kuang (2010b) found that cloud-base variations contributed negligibly to variations in the properties of updraughts by the time they reached the free troposphere in a simulation of nonprecipitating convection. These studies, and the low-level MSE distribution in our simulations, suggest that cloud-base variations are relatively unimportant in determining the properties of clouds at higher levels, providing justification for our use of a single value for temperature and specific humidity as the lower-boundary 
condition for the plume spectrum. Indeed, the correspondence between the low-level plume MSE and high percentiles of the simulated MSE distribution suggests that the one-standard-deviation perturbation used to initialize the plumes provides a reasonable estimate of the initial properties of updraughts in the simulations.

The lower panels of Figure 5 show distributions of $(\mathrm{d}, \mathrm{e}, \mathrm{f})$ buoyancy, $(\mathrm{g}, \mathrm{h}, \mathrm{i})$ vertical velocity, and $(\mathrm{j}, \mathrm{k}, \mathrm{l})$ condensed water content in the MSE-height plane. For the simulations, these properties are calculated based on hourly snapshots and then binned by MSE at each level (colours). The bin boundaries are positioned as a cubic function of MSE such that the bin width increases with increasing MSE. Results are contoured only for bins with at least 40 grid points contributing. Similar distributions are plotted for the plume spectrum by interpolating the properties of individual plumes in the spectrum to standard values of MSE and then contouring (thin black lines).

The buoyancy and vertical velocity distributions derived from the plume spectrum agree well with those derived from the simulations as a function of height and MSE. For both the plume spectrum and simulations, the buoyancy and vertical velocity increase with decreasing effective entrainment rate, and their magnitudes are similar between the plume spectrum and simulations. In addition, the plume spectrum captures the rough magnitude of the increase of the buoyancy and vertical velocity with warming. For instance, the highest percentiles of the MSE distribution have peak vertical velocities of $4-8(16-20) \mathrm{m} \mathrm{s}^{-1}$ in the coldest (warmest) simulation, and this is captured by the plume spectrum, although the precise values depend somewhat on the values of $a$ and $b$ used in Eq. (6).

Differences in the buoyancy of cloudy parcels at a given MSE are primarily related to differences in their condensed water content. The plume spectrum somewhat overestimates the amount of condensed water as a function of MSE and height, likely as a result of the simple assumption of no fallout in the plume calculation; better agreement is found if some precipitation fallout is included (not shown). The plume spectrum nonetheless exhibits an increase in the amount of condensed water as the effective entrainment rate decreases, consistent with the simulations, and it also captures the increase in condensate amount with warming among high MSE percentiles in the simulations. Thus, we argue that the assumption of no precipitation fallout in the plume calculations does not severely limit the utility of the plume spectrum as a conceptual model through which to understand the behaviour of the updraughts.

Having shown the fidelity of the plume spectrum in reproducing the buoyancy and updraught velocities for high MSE percentiles, we consider again the distribution of effective entrainment rates. As noted earlier, the 99.999th percentile of MSE has a similar effective entrainment rate through a large portion of the troposphere in all the simulations shown. Similarly, the 99.9th percentile of MSE has an effective entrainment rate of roughly $0.5 \mathrm{~km}^{-1}$ in the lower troposphere of all the simulations shown. (While the values of the entrainment rate correspond to lengthscales that are not very different from the smallest horizontal scale resolvable by the model, very similar entrainment distributions are found in the low-resolution simulations, suggesting some robustness to resolution changes.) The consistency of the effective entrainment rate for high percentiles of the MSE distribution at different surface temperatures suggests that insight may be gained by assuming the entrainment distribution among plumes remains fixed as the atmosphere warms. In other words, it may be helpful to assume that the fraction of air parcels with effective entrainment rates exceeding a given value does not vary with warming. We explore this possibility by comparing the buoyancy integral and peak updraught in the simulations to those of a plume with a fixed entrainment rate (Figure 6). As in the previous section, our measure of the buoyancy integral in the simulations is based on the profile of the 99.99th percentile of buoyancy, and the peak updraught is taken as the maximum of the 99.99th percentile vertical velocity profile, $w_{\max }$. An entrainment rate of $0.15 \mathrm{~km}^{-1}$
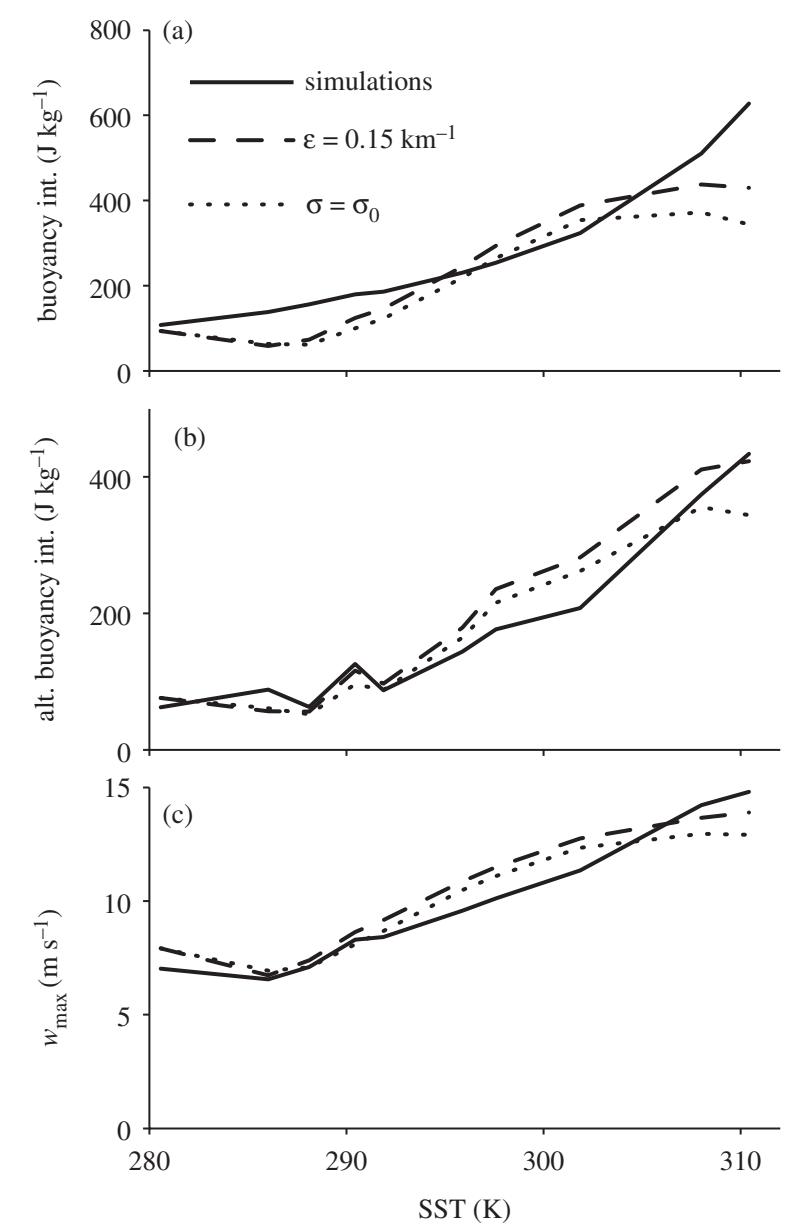

Figure 6. Buoyancy integral (based on the 99.99th percentile of buoyancy) and $w_{\max }$ (the peak of the 99.99th percentile updraught profile) for high-resolution simulations (solid) and as estimated by a plume calculation with an entrainment rate of $0.15 \mathrm{~km}^{-1}$ (dashed). (a) Buoyancy integral, (b) alternative buoyancy integral in which the upper limit of the integral is the level at which $w_{\max }$ occurs in the simulations, and (c) $w_{\max }$. Results are also shown for an alternative plume calculation in which the lower-boundary perturbations to the temperature and specific humidity are fixed to their values in the coldest simulation (dotted).

is chosen to provide a good overall fit to the simulated buoyancy integral and $w_{\max }$ although this is somewhat below the value implied for the 99.99th percentile of MSE based on Figure 5. This discrepancy may be partly because the MSE does not entirely determine buoyancy, and thus the percentiles of buoyancy and vertical velocity need not correspond to percentiles of the MSE (or effective entrainment) distribution, and the discrepancy is reduced somewhat if fallout is included in the plume calculation (not shown).

The fixed-entrainment plume roughly reproduces the magnitude of the increase in the buoyancy integral and $w_{\max }$ with warming (Figure 6(a,c)). For example, the simulated buoyancy integral increases by a factor of 6 across the simulations, while the buoyancy integral according to the fixed-entrainment plume calculation increases by a factor of 5 . Since the entrainment rate and microphysical assumptions of the plume remain fixed as the surface temperature varies, the increase in the plume estimate of the buoyancy integral is a result of changes in the plume environment, as represented by the mean profiles in the simulations, or changes in the lower-boundary condition for the plumes. The dotted lines in Figure 6 show results based on a plume calculation in which the perturbations used in the plume lower-boundary condition (i.e. $\sigma_{T}$ and $\sigma_{q}$ in Eq. (4)) are fixed to their value in the coldest simulation. As can be seen from the similarity between the dotted and dashed lines in Figure 6, changes to the lower-boundary perturbation contribute only a small fraction of the increase in updraught velocity estimated by the fixed-entrainment plume calculation. Additional plume calculations in which the relative humidity of the environment is fixed to a constant value (not 

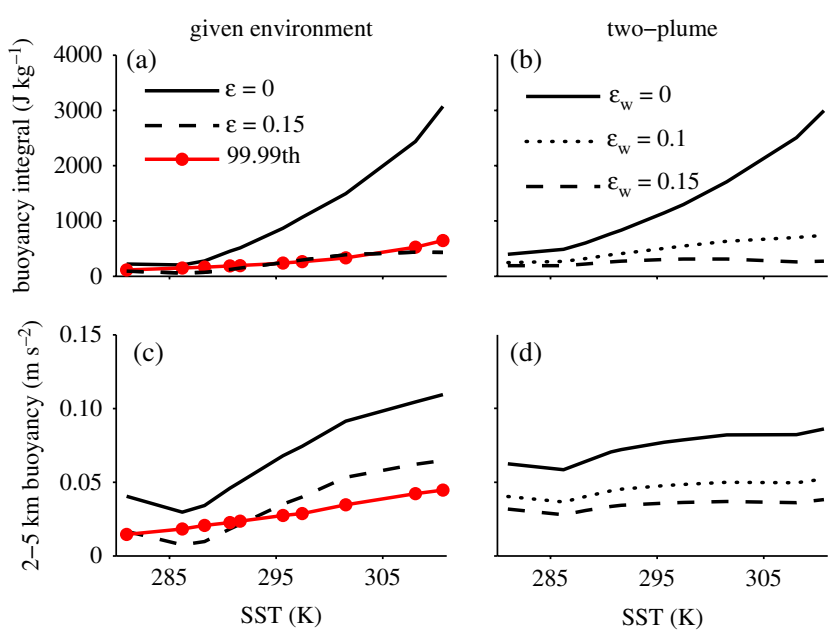

Figure 7. Estimates of $(a, b)$ buoyancy integral and $(c, d)$ mean buoyancy between 2 and $5 \mathrm{~km}$ for $(\mathrm{a}, \mathrm{c})$ the plume calculation with given environmental properties and $(b, d)$ the two-plume model. Estimates are based on undilute plumes (solid) and plumes with entrainment rates of $0.1 \mathrm{~km}^{-1}$ (dotted) and $0.15 \mathrm{~km}^{-1}$ (dashed). The buoyancy integral is calculated as the integral of the plume buoyancy up to the level at which the environment temperature is $220 \mathrm{~K}$. Also shown in (a,c) are results based on the 99.99th percentile buoyancy profile in the high-resolution simulations (red line and circles). See text for details of plume calculations.

shown) suggest that the increase in the plume estimate of the peak updraught is mostly due to changes to the mean lapse rate in the simulations with warming.

The fixed-entrainment plume estimate does not capture the detailed dependence of the simulated buoyancy integral on surface temperature (Figure 6(a)), whereas the variation in $w_{\max }$ with temperature is well captured by the fixed-entrainment plume (Figure 6(c)). The value of $w_{\max }$ given by the plume calculation is only dependent on the plume buoyancy below the level of $w_{\max }$, and better agreement between the buoyancy integrals from the plume spectrum and simulations is found if the integral is terminated at the level of $w_{\max }$ in the simulations (Figure 6(b)).

Overall, the fixed-entrainment plume results suggest that some knowledge of the behaviour of convective updraughts as a function of surface temperature can be derived from plume arguments applied to large-scale fields (cf. Del Genio et al., 2007), but the precise rate of change in updraught velocity given by such estimates should be taken with caution.

The fractional increase with warming in the buoyancy integral estimated from the plume spectrum is much larger if an undilute set of plumes is used in the calculation (Figure 7(a)). The buoyancy integral of an undilute plume increases by roughly a factor of 14 over the range of surface temperatures simulated, whereas the buoyancy integral in the simulations increases by a factor of 6 , and the buoyancy integral calculated from the plume with an entrainment rate of $0.15 \mathrm{~km}^{-1}$ increases by a factor of 5 . The CAPE increases by an even greater factor than the undilute buoyancy integral, in part because it is calculated up to the level of neutral buoyancy which is higher than the $220 \mathrm{~K}$ isotherm. The plume spectrum thus suggests that the differing scaling of CAPE and cloud buoyancy integral in the simulations is related to the effect of entrainment on even the most intense updraughts.

\section{Two-plume model}

We next investigate why the inclusion of entrainment results in a lower fractional increase in the plume estimate of the buoyancy integral. We do this by extending the zero-buoyancy plume model of SO13 to include a prediction for the buoyancy of the most intense updraughts in addition to predicting the environmental lapse rate. Given the difficulty of accurately reproducing the simulated buoyancy integral, even when the environment is given, we do not expect detailed agreement between this simple model and the simulated buoyancy profiles. Instead, we seek to understand, on the conceptual level, how entrainment in the simulations leads to a lower fractional rate of increase in the buoyancy integral with warming as compared to its undilute counterpart. In this spirit, we first solve a simplified version of the two-plume model analytically, before showing numerical results of an implementation that requires fewer approximations.

\subsection{Analytic formulation}

We represent the ensemble of convective clouds in RCE by two plumes; the first plume represents the bulk of the convective mass flux, and has a relatively strong entrainment rate $\epsilon_{\mathrm{s}}$, while the second plume represents the most intense updraughts in the ensemble with a weaker entrainment rate $\epsilon_{\mathrm{w}}$.

First we consider the strongly entraining plume with temperature $T_{\mathrm{s}}$ and moist static energy $h_{\mathrm{s}}$. Following the arguments of SO13, we neglect the buoyancy of the strongly entraining plume and assume it is exactly neutrally buoyant with respect to the environment. We can make some progress analytically if we neglect the effect of water on density and heat capacity and the contribution of ice-phase condensate to the plume MSE. Under these conditions the assumption of zero buoyancy reduces to $T_{\mathrm{s}}=T_{\mathrm{e}}$. Above the cloud base, the plume is saturated and we have that $h_{\mathrm{s}}=h_{\mathrm{s}}^{*}=h_{\mathrm{e}}^{*}$, where the asterisk refers to a variable at saturation. Substituting this expression into the plume equation (1) allows us to write an equation entirely in terms of environmental properties and the entrainment rate $\epsilon_{\mathrm{s}}$,

$$
\frac{\mathrm{d} h_{\mathrm{e}}^{*}}{\mathrm{~d} z}=-\epsilon_{\mathrm{s}}\left(h_{\mathrm{e}}^{*}-h_{\mathrm{e}}\right)
$$

This is similar to Eq. (2) of SO13.

We now consider the weakly entraining plume, with moist static energy $h_{\mathrm{w}}$, temperature $T_{\mathrm{w}}$, and entrainment rate $\epsilon_{\mathrm{w}}$. Above the cloud base, this second plume is governed by

$$
\frac{\mathrm{d} h_{\mathrm{w}}^{*}}{\mathrm{~d} z}=-\epsilon_{\mathrm{w}}\left(h_{\mathrm{w}}^{*}-h_{\mathrm{e}}\right) .
$$

Subtracting Eq. (7) from Eq. (8) and solving the resultant ordinary differential equation gives

$$
h_{\mathrm{w}}^{*}(z)-h_{\mathrm{e}}^{*}(z)=\delta \epsilon \int_{z_{\mathrm{b}}}^{z} \mathrm{e}^{-\epsilon_{\mathrm{w}}\left(z-z^{\prime}\right)}\left\{h_{\mathrm{e}}^{*}\left(z^{\prime}\right)-h_{\mathrm{e}}\left(z^{\prime}\right)\right\} \mathrm{d} z^{\prime},
$$

where $\delta \epsilon=\epsilon_{\mathrm{s}}-\epsilon_{\mathrm{w}}$ and we have assumed the plumes are identical below the level of the cloud base $z_{\mathrm{b}}$. Additionally, we have assumed $\epsilon_{\mathrm{s}}$ and $\epsilon_{\mathrm{w}}$ are constant with height in order to simplify the presentation, but we will relax this assumption in the next section.

Since $h_{\mathrm{e}}^{*}-h_{\mathrm{e}}=L_{\mathrm{v}}\left(q_{\mathrm{ve}}^{*}-q_{\mathrm{ve}}\right)$, the right-hand side of Eq. (9) depends on the saturation deficit of the free troposphere. Linearizing the Clausius-Clapeyron equation about the environment temperature, we may write an expression for the temperature excess of the weakly entraining plume,

$$
T_{\mathrm{w}}(z)-T_{\mathrm{e}}(z)=\frac{\delta \epsilon(1-\mathcal{R})}{1+\frac{L_{\mathrm{v}}^{2} q_{\mathrm{ve}}^{*}(z)}{c_{p} R_{\mathrm{v}} T_{\mathrm{e}}^{2}(z)}} \int_{z_{\mathrm{b}}}^{z} \mathrm{e}^{-\epsilon_{\mathrm{w}}\left(z-z^{\prime}\right)} \frac{L_{\mathrm{v}} q_{\mathrm{ve}}^{*}\left(z^{\prime}\right)}{c_{p}} \mathrm{~d} z^{\prime},
$$

where $\mathcal{R}$ is the relative humidity of the environment, approximated by $q_{\mathrm{ve}} / q_{\mathrm{ve}}^{*}$ and assumed constant in the vertical, and $R_{\mathrm{v}}$ is the gas constant for water vapour.

Multiplying by $g / T_{\mathrm{e}}$, Eq. (10) gives an approximate expression for the buoyancy of the weakly entraining plume, assuming the strongly entraining plume has zero buoyancy. In the limit of $\epsilon_{\mathrm{w}} \rightarrow 0$, this is identical to Eq. (4) of SO13 describing the buoyancy of undilute ascent. Here, we are interested in the case where the strongest updraughts, while having relatively low entrainment rates, are still far from undilute.

The expression (10) states that the buoyancy of the weakly entraining plume at some height $z$ is proportional to the difference 
in entrainment rates between the plumes, $\delta \epsilon$, the relative humidity deficit of the environment, $1-\mathcal{R}$, and a function of the saturation specific humidity that depends on its weighted integral below $z$. The dependence on the saturation specific humidity ensures that, for typical atmospheric temperatures, the buoyancy increases with temperature, assuming modest changes in the relative humidity of the environment and modest changes to the entrainment rates of the plumes. At very high temperatures, the denominator in Eq. (10) becomes large, and the dependence of the weakly entraining buoyancy (at a fixed height) on temperature becomes weak.

Motivated by the difference in behaviour of (undilute) CAPE and the buoyancy integral in the simulations, we next use Eq. (10) to calculate the ratio of the buoyancy in the weakly entraining plume $\left(\epsilon_{\mathrm{w}}>0\right)$ to that of an undilute plume $\left(\epsilon_{\mathrm{w}}=0\right)$. For simplicity, we approximate the dependence of the saturation specific humidity on height as an exponential,

$$
q_{\mathrm{ve}}^{*}(z)=q_{\mathrm{ve}}^{*}\left(z_{\mathrm{b}}\right) \exp \left(-\frac{z-z_{\mathrm{b}}}{H_{\mathrm{q}}}\right),
$$

where $H_{q}$ is the scale height for water vapour. In the undilute case $\left(\epsilon_{\mathrm{w}}=0\right)$, the integral in Eq. (10) may then be written

$$
\int_{z_{\mathrm{b}}}^{z} \frac{L_{\mathrm{v}} q_{\mathrm{ve}}^{*}\left(z^{\prime}\right)}{c_{\mathrm{p}}} \mathrm{d} z^{\prime}=\frac{L_{\mathrm{v}} H_{\mathrm{q}}}{c_{\mathrm{p}}}\left\{q_{\mathrm{ve}}^{*}\left(z_{\mathrm{b}}\right)-q_{\mathrm{ve}}^{*}(z)\right\} .
$$

For the weakly entraining plume we must also consider the exponential weighting function containing the entrainment rate $\epsilon_{\mathrm{w}}$. The result of the integral in Eq. (10) is then dependent on the relative magnitudes of the water vapour scale height $H_{\mathrm{q}}$ and the entrainment length-scale $\epsilon_{\mathrm{w}}^{-1}$. The water vapour scale height is of the order of $2-3 \mathrm{~km}$, while the entrainment length-scale is in the range $2-10 \mathrm{~km}$ for the buoyant plumes in Figure 5. For simplicity, we consider the case in which these two length-scales are equal; our conclusions are similar if $H_{\mathrm{q}}$ and $\epsilon_{\mathrm{w}}^{-1}$ are only of the same order rather than equal, but the resultant analytic expressions are more complicated.

If $H_{\mathrm{q}}=\epsilon_{\mathrm{w}}^{-1}$, the integral in Eq. (10) loses its exponential dependence, and we may write

$$
\int_{z_{\mathrm{b}}}^{z} \mathrm{e}^{-\epsilon_{\mathrm{w}}\left(z-z^{\prime}\right)} \frac{L_{\mathrm{v}} q_{\mathrm{ve}}^{*}\left(z^{\prime}\right)}{c_{\mathrm{p}}} \mathrm{d} z^{\prime}=\left(z-z_{\mathrm{b}}\right) \frac{L_{\mathrm{v}} q_{\mathrm{ve}}^{*}(z)}{c_{\mathrm{p}}} .
$$

Applying Eqs (11) and (12) to Eq. (10), the ratio of the buoyancy of the weakly entraining plume to the undilute buoyancy may then be written

$$
\frac{T_{\mathrm{w}}-T_{\mathrm{e}}}{T_{\mathrm{u}}-T_{\mathrm{e}}}=\frac{\delta \epsilon}{\epsilon_{\mathrm{s}}} \frac{\left(z-z_{\mathrm{b}}\right)}{H_{\mathrm{q}}} \frac{q_{\mathrm{ve}}^{*}(z)}{q_{\mathrm{ve}}^{*}\left(z_{\mathrm{b}}\right)-q_{\mathrm{ve}}^{*}(z)},
$$

where $T_{\mathrm{u}}$ is the temperature of the undilute plume, and quantities on the left-hand side are evaluated at $z$.

Consider how the buoyancy ratio given by Eq. (13) responds to warming at a fixed height in the mid-troposphere. In the above derivation, we assumed that $H_{\mathrm{q}}$ is constant in the vertical to evaluate the integral in Eq. (10). If we also make the approximation that $H_{\mathrm{q}}$ does not vary as the surface temperature increases, the buoyancy ratio given by Eq. (13) is also constant with warming. In fact, $H_{\mathrm{q}}$ does vary somewhat, both in the vertical and with surface temperature. Nevertheless, as a first approximation, we might expect that the fractional increase in the buoyancy of the weakly entraining plume at a fixed height is similar to that of the undilute buoyancy as the surface temperature increases. This is confirmed in more detailed calculations of the two-plume model outlined in the next section.

As pointed out in section 3, the top of the convecting layer remains at approximately fixed temperature as the atmosphere warms. Consider next the buoyancy ratio given by Eq. (13) in the region just below the top of the convecting layer. As the surface temperature increases, $q_{\mathrm{ve}}^{*}$ will be evaluated at roughly fixed temperature, and the only factors on the right-hand side of Eq. (13) that vary significantly are $\left(z-z_{\mathrm{b}}\right)$, which is the depth of the convecting layer, and $\left\{q_{\mathrm{ve}}^{*}\left(z_{\mathrm{b}}\right)-q_{\mathrm{ve}}^{*}(z)\right\}^{-1}$. For a roughly constant lapse rate, the depth of the convecting layer increases linearly with increasing surface temperature, whereas $q_{\mathrm{ve}}^{*}\left(z_{\mathrm{b}}\right)$ increases exponentially and at a higher fractional rate. This implies that, in the region just below the top of the convecting layer, the buoyancy ratio given by Eq. (13) decreases with warming; in other words, the fractional increase in the buoyancy of the weakly entraining plume is smaller than that of the undilute buoyancy.

The approximate results presented in this section suggest a number of conclusions about the scaling of buoyancy with temperature according to the two-plume model, assuming weak changes in the tropospheric relative humidity and the entrainment rates of the plumes:

1. The buoyancy of the weakly entraining plume increases with warming.

2. The scaling of the weakly entraining buoyancy and undilute buoyancy with temperature are similar at a fixed height in the mid-troposphere. But near the top of the convecting layer, the fractional increase in undilute buoyancy with warming is larger than that of the weakly entraining buoyancy.

3. Point (2) implies that, for the integral of the buoyancy over the convecting layer, the fractional increase is higher in the undilute case than the weakly entraining case.

These conclusions have been derived for the specific case in which the water vapour scale height is constant and equal to the entrainment length, and the plume entrainment rates $\epsilon_{\mathrm{s}}$ and $\epsilon_{\mathrm{W}}$ are constant in the vertical. We next show that the conclusions also hold for a more detailed calculation of the two-plume model in which these assumptions are not made.

\subsection{Numerical solution}

We compare results from numerical integrations of the twoplume model to results from the plume spectrum calculated in section 4 and results from the simulations. The effects of water on the density and specific heat capacity of moist air, as well as the contribution of ice to the moist static energy, are included in the plume calculations described here, unlike in the approximate treatment given in Eqs (7)-(13).

The properties of the strongly entraining plume and the environment are calculated using an identical method to that of the zero-buoyancy plume model of SO13. We set the lowerboundary condition of the strongly entraining plume to be equal to the mean properties at the lowest model level in the corresponding simulation, and we integrate the plume equations upward assuming the strongly entraining plume is exactly neutrally buoyant with respect to the environment and assuming no fallout of condensate. We use an entrainment profile given by $\epsilon_{\mathrm{s}}=0.5 / z$ and a constant environmental relative humidity of $80 \%$. The same parameters were used in SO13 to reproduce the CAPE in simulations of RCE at different surface temperatures. The entrainment profile varies inversely with height in order to crudely account for the bulk effects of many clouds detraining at different levels, since the strongly entraining plume represents much of the convective mass flux.

The weakly entraining plume, representing only the most intense updraughts, is given a constant entrainment rate. It is calculated using the environmental properties derived from the strongly entraining plume calculation as discussed above, and it is initialized at the lowest model level as in the plume spectrum; the temperature and specific humidity of the weakly entraining plume are set to one standard deviation above the mean temperature and specific humidity at the lowest model level in the simulations (Eq. (4)). (Initializing the weakly entraining plume with the 
mean properties at the lowest model level does not change the qualitative behaviour of the two-plume model.)

The two-plume model estimate of the buoyancy integral increases with warming, at least for low values of $\epsilon_{\mathrm{w}}$, and its fractional rate of increase is largest in the case where the weakly entraining plume is undilute $\left(\epsilon_{\mathrm{w}}=0\right.$; Figure $\left.7(\mathrm{~b})\right)$. On the other hand, the scaling of the mean buoyancy in the lower troposphere with temperature according to the two-plume model is similar for both the undilute and entraining cases (Figure $7(\mathrm{~d})$ ). These characteristics are broadly consistent with the behaviour of the plume spectrum with the environment taken from the simulations and with the behaviour of the simulations themselves; the fractional increase in the mean buoyancy between 2 and $5 \mathrm{~km}$ is similar for the undilute plumes, the fixed-entrainment plumes and the 99.99th percentile of buoyancy in the simulations (Figure 7(c)), while the buoyancy integral for an undilute plume increases at a much higher fractional rate than the buoyancy integral of an entraining plume, or that based on high percentiles of the buoyancy distribution (Figure $7(\mathrm{a})$ ). This suggests that the two-plume model captures some of the behaviour of the simulations, and it may be relevant for understanding the scaling of both CAPE and cloud buoyancy in the RCE simulations. According to the two-plume model, the differing scaling of entraining and undilute buoyancy integrals with temperature arises because entrainment is more effective in reducing cloud buoyancy at higher levels in the atmosphere coupled with the fact that convection deepens as the atmosphere warms.

However, there are also substantial differences between the behaviour of the two-plume model and the simulations. For example, for a value of $\epsilon_{\mathrm{w}}=0.15 \mathrm{~km}^{-1}$, which is equal to the entrainment rate used to match the simulations in the given-environment calculation, the two-plume buoyancy integral decreases with warming at high surface temperatures. It is only if a lower entrainment rate $\left(0.1 \mathrm{~km}^{-1}\right)$ is used that the two-plume model reproduces the behaviour of the simulations (Figure 7). The two-plume model is thus a useful tool for understanding the effect of entrainment on a convective ensemble, but is too crude to provide quantitatively accurate predictions of the buoyancy of clouds at different surface temperatures.

One reason for the poor quantitative agreement between the two-plume model and the simulations is that the environmental lapse rate is entirely determined by a single plume which must account for the bulk effects of all the convection in the domain. A possible alternative to the two-plume model which would address some of these deficiencies is to consider a spectrum of plumes, as in section 4, and calculate the effect of all plumes on the mean lapse rate (cf. Arakawa and Schubert, 1974). An important aspect of such a calculation would be the assumption that the entrainment distribution remains fixed as the surface temperature increases, allowing for a prediction of the updraught properties with warming. Such a detailed model of the interaction between convective clouds and their environment is beyond the scope of the present study, and we leave it to future work.

\section{Effect of changes in hydrometeor fall speed}

The plume calculations we have used to interpret the simulations do not allow for precipitation fallout. However, Parodi and Emanuel (2009) found that the fall speed of hydrometeors plays a large role in determining updraught velocities in simulations of RCE. We investigate the effect of hydrometeor fall speed on updraught velocities by conducting additional RCE simulations in which we fix the fall speed of all hydrometeors to a constant value regardless of their size or type.

For a subset of the SST (and $\mathrm{CO}_{2}$ ) values, the simulations are rerun with different imposed fall speeds in the range $1-8 \mathrm{~m} \mathrm{~s}^{-1}$, and in a lower-resolution ( $1 \mathrm{~km}$ horizontal grid-spacing), smaller-domain $(84 \times 84 \mathrm{~km})$ configuration; further details of these simulations may be found in Singh and O'Gorman (2014). Increasing the hydrometeor fall speed does have some effect

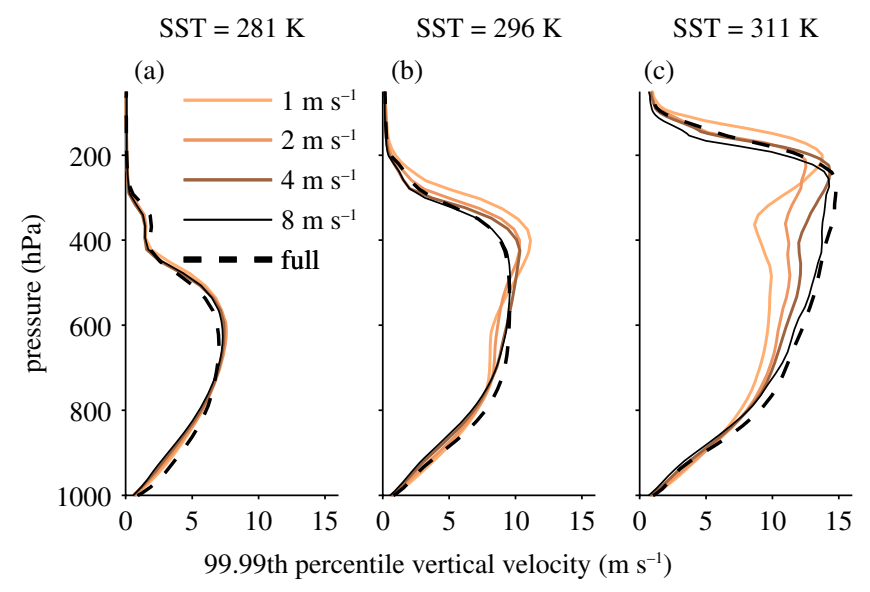

Figure 8. The 99.99th percentile of vertical velocity in full-microphysics simulations (bold black dashed) and simulations in which the fall speeds of hydrometeors are fixed to values of $1,2,4$ and $8 \mathrm{~m} \mathrm{~s}^{-1}$ as given in the legend. Simulations with imposed SSTs of (a) $281 \mathrm{~K}$, (b) $296 \mathrm{~K}$ and (c) $311 \mathrm{~K}$ are shown.

on the convective vertical velocity as measured by its 99.99th percentile (Figure 8). However, the size, and even the sign, of the effect depends on the vertical level and SST considered. The sensitivity to fall speed is largest at the highest SST; the 99.99th percentile of vertical velocity increases from roughly 10 to $12 \mathrm{~m} \mathrm{~s}^{-1}$ at pressure levels near $600 \mathrm{hPa}(\sim 5 \mathrm{~km})$ as the fall speed is increased from 1 to $8 \mathrm{~m} \mathrm{~s}^{-1}$. This sensitivity to fall speed is smaller than found in the simulations of Parodi and Emanuel (2009). Preliminary investigations indicate that a larger effect of hydrometeor fall speed is found when a simpler, warm-rain microphysics scheme is employed, as was the case in Parodi and Emanuel (2009), and this may be the cause of the discrepancy.

The average hydrometeor fall speed increases with warming in the full-microphysics simulations, mostly as a result of a switch in the predominant hydrometeor type from slower-falling snow to faster-falling rain (Singh and O'Gorman, 2014). In the cold and moderately warm simulations, the updraught velocities are insensitive to fall speed (Figure $8(\mathrm{a}, \mathrm{b})$ ), and so fall speed variations do not contribute to the increase in updraught velocities with warming. In the very warm simulations, there is some sensitivity of updraught velocities to fall speed (Figure 8(c)), but fall speeds do not increase greatly with warming at these temperatures (see Figure 1b of Singh and O'Gorman, 2014) except in the upper troposphere. We thus argue that fall speed variations are generally not an important contributor to the changes in updraught velocities in our simulations, except possibly in the upper troposphere in the very warm simulations.

\section{Summary and conclusions}

Our RCE simulations show robust increases in updraught velocities with warming over a wide range of surface temperatures, with the largest fractional increases occurring in the upper troposphere and for the highest percentile updraughts. Consistent increases in the buoyancy of clouds are also found; a roughly linear relationship exists between the vertical integral of the simulated buoyancy and the square of the peak updraught velocity. As previously documented by SO13, the CAPE also increases with warming in RCE, but the fractional increase in CAPE is considerably larger than the increase in the buoyancy integral.

The above results are understood through a series of conceptual models based on a set of entraining plumes. A plume model in which the environment is taken as the mean profile in the simulations, and the entrainment and microphysical assumptions remain fixed as the atmosphere warms, reproduces the magnitude of the simulated increase in updraught velocities with warming. This indicates that changes to the mean lapse rate are important factors leading to the increase in updraught 
velocities, notwithstanding the fact that the CAPE increases are fractionally much larger than those of the buoyancy integral as the atmosphere warms.

A generalization of the zero-buoyancy plume model introduced in SO13 to the case of two plumes was constructed to account for the differing responses to warming of CAPE and the buoyancy integral calculated from the buoyancies in the simulations. The two-plume model predicts that undilute and entraining buoyancy increase at similar fractional rates in the mid-troposphere, but because the effect of entrainment on the plume temperature increases with height and the depth of convection increases with warming, the fractional increase of entraining buoyancy is lower near the top of the convecting layer. The two-plume results are qualitatively consistent with the behaviour of the simulations, and the plume models suggest that the small value of the buoyancy integral relative to the CAPE, as well as its weaker fractional rate of increase with warming, may be explained by the effect of entrainment acting on the most intense updraughts. Since both the updraughts and the mean state are sensitive to entrainment, care must be taken when using GCM output to infer changes to cloud-scale updraughts under climate change.

The plume models used in this study do not account for precipitation fallout, but additional simulations in which the microphysical parametrization was altered to have fixed fall speeds indicate that variations in hydrometeor fall speeds play only a minor role in driving changes to the updraught velocities seen in the simulations. This insensitivity to fall speeds is in contrast to the results of Parodi and Emanuel (2009), possibly because here we focus on the effect of changes in temperature and because the microphysics scheme we use includes frozen species.

An additional factor not accounted for in the plume calculations is the role of the changing radiative cooling profile as the atmosphere warms. Robe and Emanuel (1996) found that changes to the radiative cooling profile in RCE have a weak effect on the updraught velocity, and primarily affect the area fraction over which convection occurs. These area-fraction changes could potentially affect the updraught velocity at given percentiles. For instance, Muller et al. (2011) found markedly different responses of CAPE and updraught velocities to warming in simulations of $\mathrm{RCE}$ in which the radiative cooling profile was fixed compared to cases where an interactive radiation scheme was used. The effect of changes in radiative cooling is not easily accounted for by the plume models used here.

A possible weakness of our study is that entrainment processes are important for our results but they may not be well represented in our simulations. Air parcels lifted without dilution from the boundary layer are virtually absent in our simulations whereas some previous observational studies have suggested that undilute convective updraughts are present in the tropical troposphere and play an important role in setting the thermal structure (Riehl and Malkus, 1958; Xu and Emanuel, 1989). However, more recently this interpretation of the observations has been questioned (Zipser, 2003; SO13), and modelling studies run at resolutions high enough to begin to resolve the inertial subrange have confirmed the rarity of undilute ascent in an idealized setting (Romps and Kuang, 2010a). Thus, our simulations, despite their relatively low resolution, are consistent with much of the evidence to date regarding the absence of undilute parcels in tropical deep-convective clouds.

In the tropical atmosphere, the effect of entrainment on cloud updraughts is also modulated by the degree of convective organization. For instance, the air entrained by updraughts occurring within mesoscale convective systems is likely to be considerably moister than the climatological mean, reducing the effect of entrainment on cloud buoyancy. On the other hand, atmospheric states with a higher degree of convective organization are associated with a lower mean relative humidity (Tobin et al., 2012). The simulations presented here show little evidence of mesoscale organization and do not undergo convective selfaggregation (e.g. Bretherton et al., 2005). Understanding how our results may be affected by the presence of mesoscale and large-scale circulations is an interesting avenue for future work.

Our results show that the increases in updraught velocities with warming are most pronounced in the upper troposphere, whereas convective precipitation extremes are most sensitive to lowertropospheric updraught velocities (Muller et al., 2011). As a result, the dynamical contribution to changes in precipitation extremes is relatively small, and thermodynamic and microphysical processes are dominant in driving changes to precipitation extremes with warming in our simulations (Singh and O'Gorman, 2014). On the other hand, the increase in updraught velocities could affect the production of lightning, and the strong increase in upper-tropospheric updraught velocities is associated with an increase in convective overshooting, as measured by an increase in the magnitude of negative buoyancy fluxes in the upper troposphere. Changes in convective overshooting have the potential to influence the thermodynamic structure and transport properties of the region near the tropopause (e.g. Sherwood and Dessler, 2001; Kuang and Bretherton, 2004; Jensen et al., 2007). Further work is required to determine if the changes in updraught velocities in the RCE simulations described here have implications for the tropical tropopause layer in a warmer climate.

\section{Acknowledgements}

We thank George Bryan for making available the numerical code CM1 and three anonymous reviewers for evaluating this manuscript. High-performance computing support from Yellowstone (http://n2t.net/ark:/85065/d7wd3xhc; accessed 2 May 2015) was provided by NCAR's Computational and Information Systems Laboratory, sponsored by the NSF. We acknowledge support from NSF grant AGS-1148594 and NASA ROSES grant 09-IDS09-0049.

\section{References}

Arakawa A, Schubert WH. 1974. Interaction of a cumulus cloud ensemble with the large-scale environment, Part I. J. Atmos. Sci. 31: 674-701.

Baker MB, Christian HJ, Latham J. 1995. A computational study of the relationships linking lightning frequency and other thundercloud parameters. Q. J. R. Meteorol. Soc. 121: 1525-1548.

Braun SA, Tao WK. 2000. Sensitivity of high-resolution simulations of hurricane Bob (1991) to planetary boundary-layer parameterizations. Mon. Weather Rev. 128: 3941-3961.

Bretherton CS, McCaa JR, Grenier H. 2004. A new parameterization for shallow cumulus convection and its application to marine subtropical cloud-topped boundary layers. Part I: Description and 1D results. Mon. Weather Rev. 132: 864-882.

Bretherton CS, Blossey PN, Khairoutdinov M. 2005. An energy-balance analysis of deep convective self-aggregation above uniform SST. J. Atmos. Sci. 62: 4273-4292.

Bryan GH, Fritsch JM. 2002. A benchmark simulation for moist nonhydrostatic numerical models. Mon. Weather Rev. 130: 2917-2928.

Bryan GH, Rotunno R. 2009. The maximum intensity of tropical cyclones in axisymmetric numerical model simulations. Mon. Weather Rev. 137: 1770-1789.

Cronin TW, Emanuel KA. 2013. The climate time scale in the approach to radiative-convective equilibrium. J. Adv. Model. Earth Syst. 5: 843-849.

Del Genio AD, Kovari W, Yao MS, Jonas J. 2005. Cumulus microphysics and climate sensitivity. J. Clim. 18: 2376-2387.

Del Genio AD, Yao MS, Jonas J. 2007. Will moist convection be stronger in a warmer climate? Geophys. Res. Lett. 34: L16703, doi: 10.1029/2007GL030525.

Emanuel KA, Bister M. 1996. Moist convective velocity and buoyancy scales. J. Atmos. Sci. 53: 3276-3285.

Fasullo J. 2012. A mechanism for land-ocean contrasts in global monsoon trends in a warming climate. Clim. Dyn. 39: 1137-1147.

Hartmann DL, Larson K. 2002. An important constraint on tropical cloudclimate feedback. Geophys. Res. Lett. 29: 1951, doi: 10.1029/2002GL015835.

Jensen EJ, Ackerman AS, Smith JA. 2007. Can overshooting convection dehydrate the tropical tropopause layer? J. Geophys. Res. 112: D11209, doi: 10.1029/2006JD007943.

Khairoutdinov M, Emanuel K. 2013. Rotating radiative-convective equilibrium simulated by a cloud-resolving model. J. Adv. Model. Earth Syst. 5: 816-825, doi: 10.1002/2013MS000253.

Kuang Z, Bretherton CS. 2004. Convective influence on the heat balance of the tropical tropopause layer: A cloud-resolving model study. J. Atmos. Sci. 61: 2919-2927. 
Kuang Z, Bretherton CS. 2006. A mass-flux scheme view of a high-resolution simulation of a transition from shallow to deep cumulus convection. J. Atmos. Sci. 63: 1895-1909.

Kuang Z, Hartmann DL. 2007. Testing the fixed anvil temperature hypothesis in a cloud-resolving model. J. Clim. 20: 2051-2057.

Lin C, Arakawa A. 1997. The macroscopic entrainment processes of simulated cumulus ensemble. Part II: Testing the entraining-plume models. J. Atmos. Sci. 54: 1044-1053.

Lin YL, Farley RD, Orville HD. 1983. Bulk parameterization of the snow field in a cloud model. J. Clim. Appl. Meteorol. 22: 1065-1092.

Muller CJ, O'Gorman PA, Back LE. 2011. Intensification of precipitation extremes with warming in a cloud-resolving model. J. Clim. 24: 2784-2800.

Paluch IR. 1979. The entrainment mechanism in Colorado cumuli. J. Atmos. Sci. 36: 2467-2478.

Parodi A, Emanuel K. 2009. A theory for buoyancy and velocity scales in deep moist convection. J. Atmos. Sci. 66: 3449-3463.

Pauluis O, Held IM. 2002. Entropy budget of an atmosphere in radiativeconvective equilibrium. Part I: Maximum work and frictional dissipation. J. Atmos. Sci. 59: 125-139.

Pauluis OM, Mrowiec AA. 2013. Isentropic analysis of convective motions. J. Atmos. Sci. 70: 3673-3688.

Raymond DJ, Blyth AM. 1986. A stochastic mixing model for non-precipitating cumulus clouds. J. Atmos. Sci. 43: 2708-2718.

Rennó NO, Ingersoll AP. 1996. Natural convection as a heat engine: A theory for CAPE. J. Atmos. Sci. 53: 572-585.

Riehl H, Malkus JS. 1958. On the heat balance in the equatorial trough zone. Geophysica 6: 503-538.

Robe FR, Emanuel KA. 1996. Moist convective scaling: Some inferences from three-dimensional cloud ensemble simulations. J. Atmos. Sci. 53: 3265-3275.

Romps DM. 2011. Response of tropical precipitation to global warming. J. Atmos. Sci. 68: 123-138.

Romps DM, Kuang Z. 2010a. Do undiluted convective plumes exist in the upper tropical troposphere? J. Atmos. Sci. 67: 468-484.
Romps DM, Kuang Z. 2010b. Nature versus nurture in shallow convection. J. Atmos. Sci. 67: 1655-1666.

de Roode SR, Siebesma AP, Jonker HJJ, de Voogd Y. 2012. Parameterization of the vertical velocity equation for shallow cumulus clouds. Mon. Weather Rev. 140: 2424-2436.

Sherwood SC, Dessler AE. 2001. A model for transport across the tropical tropopause. J. Atmos. Sci. 58: 765-779.

Singh MS. 2014. 'The response of moist convection and the atmospheric general circulation to climate warming', $\mathrm{PhD}$ thesis. Massachusetts Institute of Technology: Cambridge, MA.

Singh MS, O'Gorman PA. 2012. Upward shift of the atmospheric general circulation under global warming: Theory and simulations. J. Clim. 25 8259-8276.

Singh MS, O'Gorman PA. 2013. Influence of entrainment on the thermal stratication in simulations of radiative-convective equilibrium. Geophys. Res. Lett. 40: 4398-4403, doi: 10.1002/grl.50796.

Singh MS, O'Gorman PA. 2014. Influence of microphysics on the scaling of precipitation extremes with temperature. Geophys. Res. Lett. 41: 6037-6044, doi: 10.1002/2014GL061222.

Sobel AH, Camargo SJ. 2011. Projected future changes in tropical summer climate. J. Clim. 24: 473-487.

Tiedtke M. 1989. A comprehensive mass flux scheme for cumulus parameterization in large-scale models. Mon. Weather Rev. 117: 1779-1800.

Tobin I, Bony S, Roca R. 2012. Observational evidence for relationships between the degree of aggregation of deep convection, water vapor, surface fluxes, and radiation. J. Clim. 25: 6885-6904.

Tompkins AM, Craig GC. 1999. Sensitivity of tropical convection to sea-surface temperature in the absence of large-scale flow. J. Clim. 12: 462-476.

Wicker LJ, Skamarock WC. 2002. Time-splitting methods for elastic models using forward time schemes. Mon. Weather Rev. 130: 2088-2097.

$\mathrm{Xu}$ KM, Emanuel KA. 1989. Is the tropical atmosphere conditionally unstable? Mon. Weather Rev. 117: 1471-1479.

Zipser EJ. 2003. Some views on 'hot towers' after 50 years of tropical field programs and two years of TRMM data. Meteorol. Monogr. 29: 49-58. 